\title{
Is iliac autogenous graft augmentation in medial open wedge high tibial osteotomies superior to no augmentation in terms of bone healing?
}

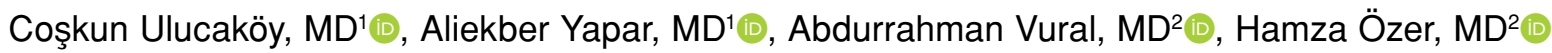 \\ 'Department of Orthopedics and Traumatology, Ankara Oncology Training and Research Hospital, Ankara, Turkey \\ ${ }^{2}$ Department of Orthopedics and Traumatology, Gazi University Faculty of Medicine, Ankara, Turkey
}

Osteoarthritis (OA) is a progressive and debilitating condition. ${ }^{[1,2]}$ Varus deformity of knee joint increases the risk of progression of medial compartment OA. High tibial osteotomy (HTO) is a well-established surgical technique for individuals with medial OA and varus deformity. ${ }^{[3]}$ The aim is to change the load distribution across the knee from the diseased medial part to the healthy lateral part in order to reduce pain, slow the degenerative process and delay the requirements for total knee replacement. ${ }^{[4]}$ Osteotomy of the proximal tibia is becoming popular as compartmental OA of the knee is increasing in younger patients with malalignment of the joint. Open wedge high tibial osteotomy (OWHTO) on the medial side has been described as an effective surgical procedure in the treatment of medial compartmental OA. ${ }^{[5,6]}$ Prevention of patella alta, easy correction of the deformity and no need for fibular osteotomy are the described advantages of medial sided osteotomy. Grafting the osteotomy site for quicker healing either with biologic resources or bone substitutes

Received: January 08, 2020

Accepted: March 08, 2020

Published online: June 18, 2020

Correspondence: Coşkun Ulucaköy, MD. Dr. Abdurrahman Yurtaslan Ankara Onkoloji Eğitim ve Araștırma Hastanesi Ortopedi ve Travmatoloji Kliniği, 06430 Yenimahalle, Ankara, Türkiye.

E-mail: coskunulucakoy@gmail.com

Doi: $10.5606 /$ ehc. 2020.73408

Citation: Ulucaköy C, Yapar A, Vural A, Özer H. Is iliac autogenous graft augmentation in medial open wedge high tibial osteotomies superior to no augmentation in terms of bone healing?. Jt Dis Relat Surg 2020;31(2):360-366.

\section{ABSTRACT}

Objectives: This study aims to investigate if iliac autogenous graft augmentation in medial open wedge high tibial osteotomies (OWHTOs) is superior to no augmentation in terms of bone healing.

Patients and methods: Twenty-five patients (14 males, 11 females; mean age $40.9 \pm 4.0$ years; range, 33 to 48 years) with medial compartmental osteoarthritis of knee joint who underwent high tibial osteotomy with medial open wedge between January 2016 and December 2018 were included in this retrospective study. Twelve of the operated knees were the right knee. Graft was used in 13 patients (52\%). Data including age, gender, body mass index (BMI), direction, follow-up period, union, Lysholm and International Knee Documentation Committee (IKDC) scores, pre- and postoperative femoral tibial angles (FTAs) and posterior tibial slopes were evaluated.

Results: The mean BMI was $26.4 \pm 1.9$ (range, 22.0 to 30.0 ). Only $48 \%$ of the patients were smoking. The mean follow-up period was $28.6 \pm 5.3$ months (range, 24 to 38 months). No statistically significant difference was found between the grafted and nongrafted groups in terms of age, BMI, follow-up time, gender, side and smoking status $(\mathrm{p}>0.05)$ There was no statistically significant difference between two groups in terms of pre- and postoperative Lysholm scores, pre- and postoperative IKDC scores, or pre- and postoperative FTA values $(\mathrm{p}>0.050)$.

Conclusion: Iliac autogenous graft augmentation in medial OWHTO has no effect on union but shortens the union time. Preoperative high varus degree adversely affects union. Therefore, routine use of iliac crest autograft is not recommended.

Keywords: High tibial osteotomy, iliac autogenous graft, union.

is controversial; however, locking plates provide a reliable implant construct until osseous healing is completed. In this study, we aimed to investigate if iliac autogenous graft augmentation in medial OWHTOs is superior to no augmentation in terms of bone healing. 


\section{PATIENTS AND METHODS}

A retrospective analysis was performed at the University of Gazi, Department of Orthopaedics and Traumatology. Patients with medial compartmental OA of knee joint were included in the study. Medial OWHTOs were performed between January 2016 and December 2018 in 42 patients. TomoFix (DePuy Synthes, NJ, USA) locking tibial osteotomy plates were used for fixation of the tibias. The patients were assessed clinically and radiographically.

Twenty-five patients (14 males, 11 females; mean age $40.9 \pm 4.0$ years; range, 33 to 48 years) were included in the study. Eleven patients with a follow-up period of less than two years, four patients without follow-up, and two patients who had previous knee surgery were excluded from the study. Data including age, gender, body mass index (BMI), side, follow-up duration, bone union, Lysholm and International Knee Documentation Committee (IKDC) scores, pre- and postoperative femorotibial angles (FTAs) and posterior tibial slopes (PTSs) were recorded. Complications such as infection, deep vein thrombosis, wound problems and loss of correction were recorded. Factors which may negatively affect healing such as smoking and osteoporosis were also recorded. The indications for HTO were KellgrenLawrence grade III-IV medial compartment OA with varus deformity, age $\leq 60$ years, tibiofemoral subluxation b10 mm, mechanical FTA b20 ${ }^{\circ}$, flexion contracture $\mathrm{b} 10^{\circ}$, and range of flexion $\mathrm{N} 90^{\circ}$. The study protocol was approved by the Dr. Abdurrahman Yurtaslan Oncology Hospital Ethics Committee on 04 September 2019 (decision number: 2019-08/363). A written informed consent was obtained from each patient. The study was conducted in accordance with the principles of the Declaration of Helsinki.

Patients were operated under general anesthesia. Tourniquet was applied to the thigh and $2 \mathrm{~g}$ of cefazolin sodium was given intravenously for the prophylaxis of infection. All patients were placed supine on the radiolucent operation table. Double incisions were used while approaching to proximal tibia. The first oblique incision was used to reach double osteotomy sites for the proximal tibia where second longitudinal incision was created over the tibia to reach the tibial shaft. An oblique osteotomy starting just above the pes anserine and aiming to the fibular head was performed. Second osteotomy was performed just behind the tibial tubercle while osteotomy planes bisected at least at $110^{\circ}$ each other. Gradual opening at the osteotomy site was performed with chisels and correction in frontal plane was controlled under fluoroscopy. Lateral cortex of the tibia was left intact while a plastic deformation was achieved during gradual opening at the osteotomy site. A TomoFix (DePuy Synthes, Johnson \& Johnson, NJ, USA) locking osteotomy plate for tibia was applied for internal fixation of the tibia. Screws were placed in the proximal metaphyseal part of the osteotomy site in locking fashion while lag screw principle was applied to lateralize the distal osteotomized tibial segment and compress lateral cortical hinge at the tibia. After placing the remaining screws on the tibia in the form of locking, the first screw, which was placed on the distal segment with the principle of lag screw, was removed.

In group A, autografts harvested from the iliac crest were placed at the osteotomy site in 13 patients. In group B, no graft was placed in 12 patients. Local infiltration anesthesia with bupivacaine applied percutaneously while no suction Hemovac drain was placed in any patient. Rivaroxaban (Xarelto ${ }^{\circledR}$, Bayer Schering Pharma AG, Berlin, Germany) $10 \mathrm{mg}$ daily was given for 10 days for the prophylaxis of deep vein thrombosis.

Patients were encouraged to partial weight-bearing with crutches on the first postoperative day. Isometric quadriceps exercises were allowed and passive/active knee exercises were allowed to gain full range of motion.

Patients were advised to use crutches until healing was confirmed via the operating surgeon. After the operation, the first radiologic evaluation was performed at six weeks. Full-length weightbearing double limb anteroposterior (AP) radiograph was taken at the final follow-up to measure FTA. Lateral radiograph was also taken for measurement of PTS. Alignment and correction were measured and healing at the osteotomy site was checked. Full consolidation of the osteotomy site was accepted while no radiolucent lines were recognizable in AP or medial/lateral planes. Union was evaluated by computed tomography (CT). Clinical evaluation was performed with Lysholm and IKDC tests pre- and postoperatively.

\section{Statistical analysis}

Statistical analysises were performed using the IBM SPSS Statistics for Windows, version 22.0 software (IBM Corp., Armonk, NY, USA). Categorical variables were given as numbers and percentages, and continuous variables were presented with mean \pm standard deviation and median (minimummaximum values) for descriptive analyses. Continuity correction chi-square test was used for comparison of categorical variables between groups. The conformity 
of continuous variables to normal distribution was evaluated using visual (histogram and probability graphs) and analytical methods (KolmogorovSmirnov/Shapiro-Wilk tests). Mann-Whitney U test was used for comparison of data sets which were not normally distributed for the variables. Wilcoxon signed-rank test was used for pre- and postoperative comparison of data sets which were not normally distributed for the variables. $\mathrm{P}$ value $<0.05$ was considered statistically significant.

\section{RESULTS}

The mean BMI was $26.4 \pm 1.9$ (range, 22.0 to 30.0 ) and only $48 \%$ of the patients were smoking. The mean follow-up period was $28.6 \pm 5.3$ months (range, 24 to 38 months). Twelve of the operated knees were the right knee (Table I). Graft was used in 13 (52\%) patients. No statistically significant difference was found between the grafted and non-grafted groups in terms of age, BMI, follow-up time, gender, side and smoking status $(\mathrm{p}>0.05)$ (Table I).

Table II shows functional scores and patients' FTAs. There was no statistically significant difference between two groups in terms of pre- and postoperative Lysholm scores, pre- and postoperative IKDC scores, or pre- and postoperative FTA values $(\mathrm{p}>0.050)$.

Table III shows the union of the osteotomy site in the postoperative period. While $24 \%$ of the patients had union in the osteotomy area at the sixth postoperative week, $96 \%$ had union in the third month and all of the osteotomy areas had union in the first year. In osteotomies with graft, union was observed in six patients at sixth week and in the remaining seven patients at third month while there was no union in the patients without graft at sixth week (Figure 1). Bone union times were significantly shorter in grafted patients than in nongrafted patients $(\mathrm{p}=0.006)$.

When the pre- and postoperative Lysholm, IKDC, and FTA values were evaluated separately in both grafted and non-grafted groups, it was found that postoperative Lysholm and IKDC scores increased significantly in both groups $(\mathrm{p}<0.050)$ (Table IV). There was a significant decrease in FTA values postoperatively compared to preoperative period $(\mathrm{p}<0.050)$.

The relationship between union time and BMI, preoperative Lysholm, IKDC and FTA scores was investigated by Spearman correlation analysis. Only a statistically significant negative moderate correlation was found between preoperative FTA values and union time $(\mathrm{r}=-0.422 ; \mathrm{p}=0.036)$. In other words,

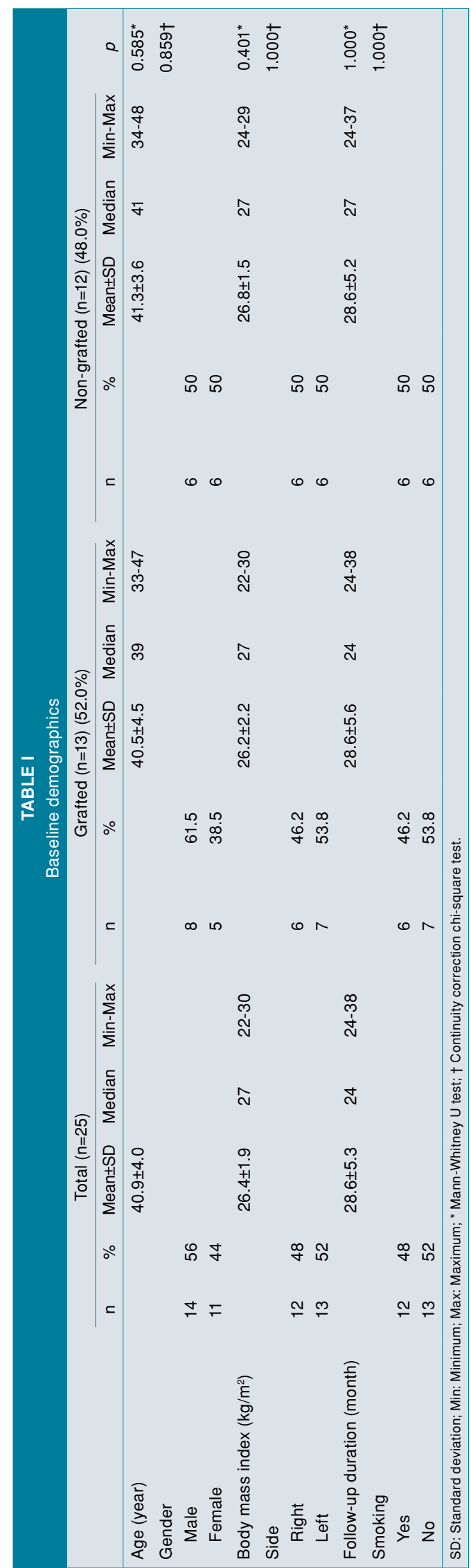



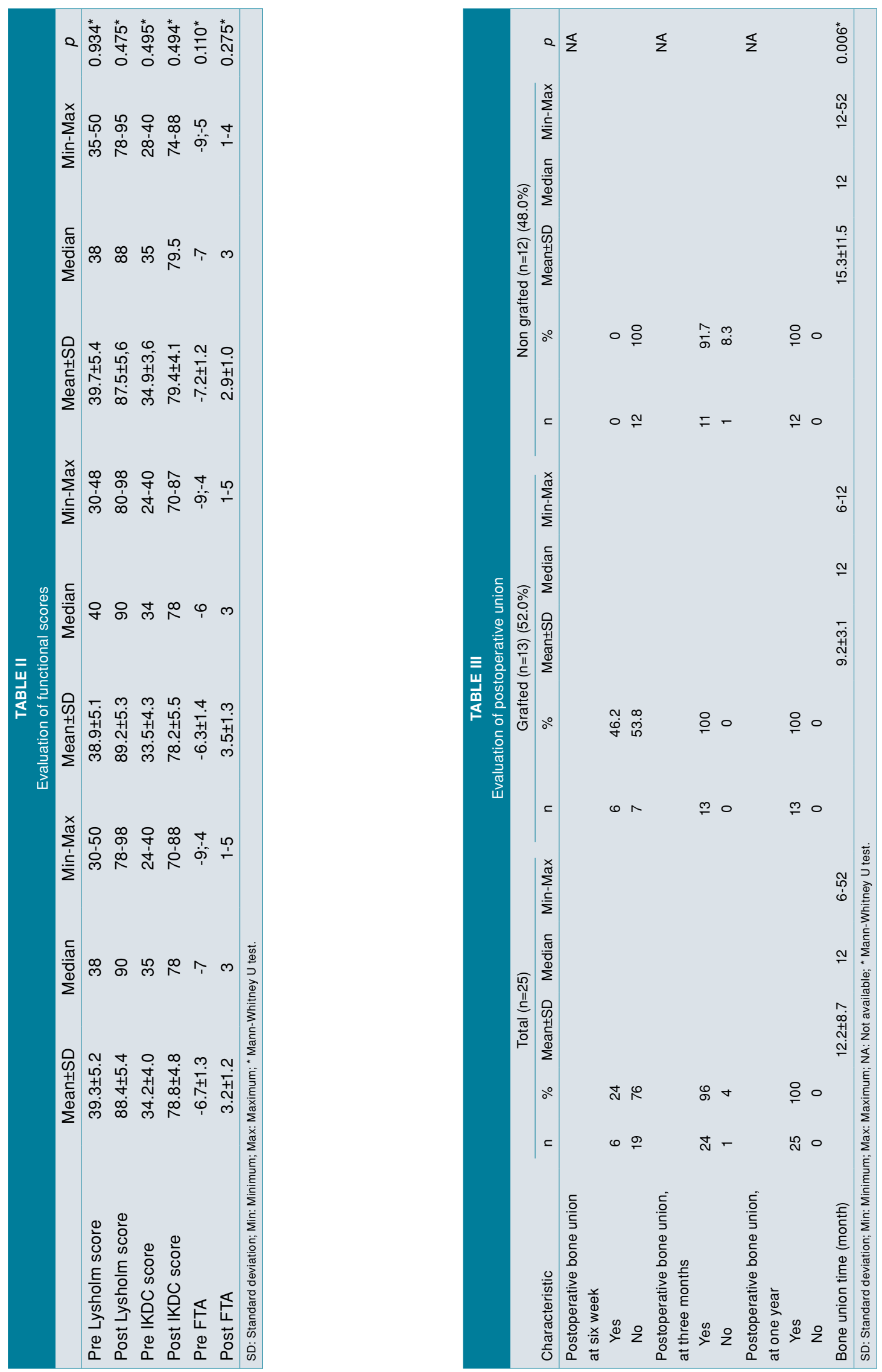


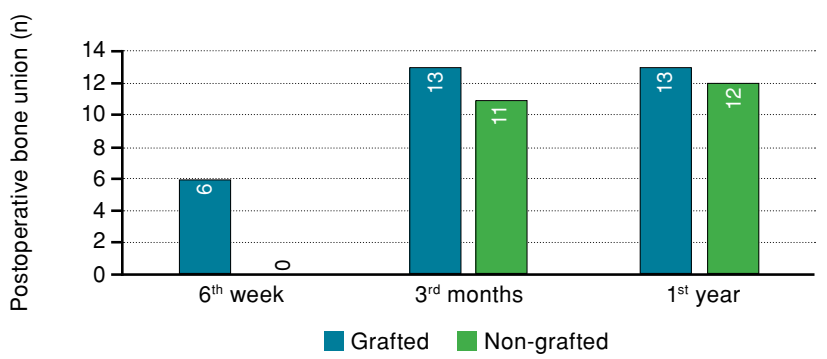

FIGURE 1. Postoperative union time of bones.

if preoperative FTA values were low in patients who underwent osteotomy, bone union times were significantly longer.

In this study, the factors affecting bone union time after osteotomy were examined by linear regression analysis in Table V. When the variables $p<0.2$ in the univariate linear regression analysis were included in the multivariate linear regression analysis model (age and gender adjusted) and examined, it was found that the union time and only preoperative FTA score were correlated alone, and having low FTA values was a risk factor that prolonged the bone union time $(\mathrm{B}=-2.701 ; \mathrm{p}=0.050)$.

Multivariate analyses confirmed the direct independent associations between bone union time and preoperative FTA observed at the univariate analyses. After adjustment for age and gender, bone union time in patients who had low preoperative FTA values remained higher $(B=-2.701 ; p=0.05)$, indicating that having low FTA values is a risk factor for longer bone union time.

\section{DISCUSSION}

Although many treatment options have been described for medial compartment arthrosis, HTO is still the most effective method, particularly for young to middle-aged patients who are not eligible for arthroplasty procedures. ${ }^{[7,8]}$ Studies reveal survivorships with good and very good results after 10 years in 63 to $96 \%$ of patients. ${ }^{[9]}$

There is no consensus on the use of grafts in medial OWHTO. The most important findings of the

\begin{tabular}{|c|c|c|c|c|c|c|c|}
\hline \multicolumn{8}{|c|}{$\begin{array}{c}\text { TABLE IV } \\
\text { Pre- and postoperative patient-reported outcome scores }\end{array}$} \\
\hline & \multicolumn{3}{|c|}{ Preoperative } & \multicolumn{3}{|c|}{ Postoperative } & \multirow[b]{2}{*}{$p$} \\
\hline & Mean $\pm S D$ & Median & Min-Max & Mean $\pm S D$ & Median & Min-Max & \\
\hline Lysholm score & $38.9 \pm 5.2$ & 40 & $30-48$ & $89.2 \pm 5.3$ & 90 & $80-98$ & $0.001^{*}$ \\
\hline IKDC score & $33.5 \pm 4.3$ & 34 & $24-40$ & $78.2 \pm 5.5$ & 78 & $70-87$ & $0.001^{*}$ \\
\hline Femoral tibial angle & $-6.3 \pm 1.4$ & -6 & $-9 ;-4$ & $3.5 \pm 1.3$ & 3 & $1-5$ & $0.001^{*}$ \\
\hline Lysholm score & $39.7 \pm 5.4$ & 38 & $35-50$ & $87.5 \pm 5.6$ & 88 & $78-95$ & $0.002^{*}$ \\
\hline IKDC score & $34.9 \pm 3.6$ & 35 & $28-40$ & $79.4 \pm 4.1$ & 79.5 & $74-88$ & $0.002^{*}$ \\
\hline Femoral tibial angle & $-7.2 \pm 1.2$ & -7 & $-9 ;-5$ & $2.9 \pm 1.0$ & 3 & $1-4$ & $0.002^{*}$ \\
\hline
\end{tabular}

\begin{tabular}{|c|c|c|c|c|}
\hline \multicolumn{5}{|c|}{$\begin{array}{l}\text { TABLE V } \\
\text { Linear regression analysis to bone union time }\end{array}$} \\
\hline & \multicolumn{2}{|c|}{ Univariate } & \multicolumn{2}{|c|}{ Multivariate } \\
\hline & B & $p$ & Adjusted B & $p$ \\
\hline $\mathrm{Age}^{*}$ & 0.605 & 0.174 & 0.466 & 0.252 \\
\hline Gender (male vs. female) & 2.234 & 0.535 & - & - \\
\hline Body mass index & -0.021 & 0.983 & - & - \\
\hline Side (right vs. left) & -4.154 & 0.241 & - & - \\
\hline Smoking (yes vs. no) & 4.179 & 0.238 & - & - \\
\hline Grafted (vs. non Grafted)* & -6.103 & 0.079 & -4.018 & 0.224 \\
\hline Pre Lysholm score & 0.066 & 0.851 & - & - \\
\hline Pre IKDC score & -0.199 & 0.667 & - & - \\
\hline Pre femoral tibial angle* & -2.861 & 0.028 & -2.701 & 0.050 \\
\hline
\end{tabular}


present study were that the use of grafts shortened the union time but did not change the union and that larger correction angles require more time to heal with and without iliac crest autograft.

Delayed bone healing or nonunion and potential loss of correction are serious complications of OWHTO ${ }^{[10]}$ Autograft union potential is higher than allograft and synthetic grafts, due to its osteogenic, osteoinductive, and osteoconductive capacities. ${ }^{[1,12]}$ However, controversy continues about the superiority of OWHTO with autograft to no graft. ${ }^{[10-12]}$ In their study with 46 patients who underwent OWHTO, Zorzi et al. ${ }^{[13]}$ reported that there was a total union in the $13^{\text {th }}$ month in both grafted and non-grafted groups, and that there was no statistically significant difference between the two groups. However, they did not use CT to assess union. Aryee et al. ${ }^{[14]}$ suggested that autograft should be used in patients with heavy smoking, obesity, and an opening gap of more than $10 \mathrm{~mm}$, which they describe as high risk. In the present study, we evaluated union by tomography. We found that autograft had no advantage in normal patient population.

Autograft has major disadvantages such as prolonged pain due to harvesting morbidity. ${ }^{[15]}$ Although synthetic bone grafts (SBGs) seem to be suitable for this, they have several disadvantages such as soft tissue irritation and infection, lack of primary stability, poor remodeling, and incorporation into bone. ${ }^{[16]}$ In addition, some authors reported worse results after OWHTO using a SBG due to delayed union or nonunion. ${ }^{[17]}$ We do not recommend using the graft routine in order not to create additional morbidity, since we did not find any statistical difference in terms of union between the groups.

Luites et al. ${ }^{[18]}$ presented the functional results of HTO in 42 patients. They reported that the Lysholm score increased from 63 to 86 after a 24-month follow-up. Hoell et al. ${ }^{[19]}$ reported a statistically significant increase in pre- and postoperative Lysholm scores in 108 patients after 24 months. Similar to the studies of Luites et al., ${ }^{[18]}$ and Hoell et al. ${ }^{[19]}$ the Lysholm score increased significantly both in grafted and non-grafted groups. Jung et al. ${ }^{[20]}$ stated that postoperative Lysholm and IKDC scores increased statistically significantly in 137 patients in 2019. In the present study, preoperative IKDC scores were 33 and 34 and postoperative IKDC scores were 78 and 79 in grafted and non-grafted groups, respectively. There was a statistically significant difference. Fucentese et al., ${ }^{[21]}$ in 40 prospective patients with medial OWHTO, did not find any significant difference between the functional results in the grafted and non-grafted groups, so they did not recommend the routine use of graft. We do not recommend the routine use of graft in order not to create additional morbidity since we did not find any statistical difference in terms of functional scores between the groups.

Articles have been published suggesting that the higher degree of preoperative varus requires more correction and leads to more complications. ${ }^{[22]}$ In their prospective study with 40 patients who underwent medial OWHTO, Fucentese et al. ${ }^{[21]}$ found shorter union time of the group using autograft and concluded that preoperative high varus delayed union. In the present study, union time of the autograft group was statistically significantly shorter and preoperative high varus angle was the only factor that delayed union. Many authors agree that keeping the valgus between $3^{\circ}$ and $5^{\circ}$ after correction will result in the longest survival and the least complications. ${ }^{[2]}$ Luites et al. ${ }^{[18]}$ reported that FTA increased significantly from $5^{\circ}$ varus to postoperative $3.4^{\circ}$ valgus. In this study, preoperative varus degrees were $6.3^{\circ}$ and $7.1^{\circ}$, and postoperative valgus degrees were $3.4^{\circ}$ and $2.9^{\circ}$ in grafted and non-grafted groups, respectively. We found a statistically significant difference between the change in pre- and postoperative FTA degrees in both groups.

The present study had certain limitations. Like most studies on the subject, it had a retrospective design. Furthermore, the sample sizes were small in both groups. Moreover, the results of the study do not reveal a standardized degree of preoperative varus for graft using in HTO.

In conclusion, iliac autogenous graft augmentation in medial OWHTO has no effect on union but shortens the union time. Preoperative high varus degree adversely affects union. Therefore, routine use of iliac crest autograft is not recommended.

\section{Declaration of conflicting interests}

The authors declared no conflicts of interest with respect to the authorship and/or publication of this article.

\section{Funding}

The authors received no financial support for the research and/or authorship of this article.

\section{REFERENCES}

1. Tokgöz MA, Atik OŞ, Esendağlı G, Öğüt B, Bozkurt HH. Is it possible that the pathogenesis of osteoarthritis could start with subchondral trabecular bone loss like osteoporosis? Eklem Hastalik Cerrahisi 2018;29:152-8.

2. Atik OŞ, Sezgin EA, Tepedelenlioğlu HE. The role of biomarkers in osteoarthritis and osteoporosis for early 
diagnosis and monitoring prognosis. Eklem Hastalik Cerrahisi 2019;30:175-6.

3. Petersen W, Metzlaff S. Open wedge high tibial osteotomy (HTO) versus mobile bearing unicondylar medial joint replacement: five years results. Arch Orthop Trauma Surg 2016;136:983-9.

4. Lobenhoffer P, De Simoni C, Staubli A. Open-wedge hightibial osteotomy with rigid plate fixation. Tech Knee Surg 2002;1:93-105.

5. Takeuchi R, Umemoto Y, Aratake M, Bito H, Saito I, Kumagai $\mathrm{K}$, et al. A mid term comparison of open wedge high tibial osteotomy vs unicompartmental knee arthroplasty for medial compartment osteoarthritis of the knee. J Orthop Surg Res 2010;5:65.

6. Santoso MB, Wu L. Unicompartmental knee arthroplasty, is it superior to high tibial osteotomy in treating unicompartmental osteoarthritis? A meta-analysis and systemic review. J Orthop Surg Res 2017;12:50.

7. Feeley BT, Gallo RA, Sherman S, Williams RJ. Management of osteoarthritis of the knee in the active patient. J Am Acad Orthop Surg 2010;18:406-16.

8. Prodromos CC, Amendola A, Jakob RP. High tibial osteotomy: Indications, techniques. Instr Course Lect 2015;64:555-65.

9. Paley D. Principles of deformity correction. Berlin: Springer; 2014.

10. Martin R, Birmingham TB, Willits K, Litchfield R, Lebel ME, Giffin JR. Adverse event rates and classifications in medial opening wedge high tibial osteotomy. Am J Sports Med 2014;42:1118-26.

11. Lash NJ, Feller JA, Batty LM, Wasiak J, Richmond AK. Bone grafts and bone substitutes for opening-wedge osteotomies of the knee: a systematic review. Arthroscopy 2015;31:720-30.

12. Harris JD, McNeilan R, Siston RA, Flanigan DC. Survival and clinical outcome of isolated high tibial osteotomy and combined biological knee reconstruction. Knee 2013;20:154-61.

13. Zorzi AR, da Silva HG, Muszkat C, Marques LC, Cliquet A Jr, de Miranda JB. Opening-wedge high tibial osteotomy with and without bone graft. Artif Organs 2011;35:301-7.

14. Aryee S, Imhoff AB, Rose T, Tischer T. Do we need synthetic osteotomy augmentation materials for opening-wedge high tibial osteotomy. Biomaterials 2008;29:3497-502.

15. Lee JS, Park YJ, Wang L, Chang YS, Shetty GM, Nha KW. Modified Iliac Crest Reconstruction with Bone Cement for Reduction of Donor Site Pain and Morbidity after Open Wedge High Tibial Osteotomy: A Prospective Study. Knee Surg Relat Res 2016;28:277-82.

16. Pornrattanamaneewong C, Numkanisorn S, Chareancholvanich $\mathrm{K}$, Harnroongroj T. A retrospective analysis of medial opening wedge high tibial osteotomy for varus osteoarthritic knee. Indian J Orthop 2012;46:455-61.

17. Gouin F, Yaouanc F, Waast D, Melchior B, Delecrin J, Passuti N. Open wedge high tibial osteotomies: Calciumphosphate ceramic spacer versus autologous bonegraft. Orthop Traumatol Surg Res 2010;96:637-45.

18. Luites JW, Brinkman JM, Wymenga AB, van Heerwaarden RJ. Fixation stability of opening- versus closing-wedge high tibial osteotomy: a randomised clinical trial using radiostereometry. J Bone Joint Surg [Br] 2009;91:1459-65.

19. Hoell S, Suttmoeller J, Stoll V, Fuchs S, Gosheger G. The high tibial osteotomy, open versus closed wedge, a comparison of methods in 108 patients. Arch Orthop Trauma Surg 2005;125:638-43.

20. Jung WH, Takeuchi R, Kim DH, Nag R. Faster union rate and better clinical outcomes using autologous bone graft after medial opening wedge high tibial osteotomy. Knee Surgery, Sports Traumatology. Arthroscopy 2019;28:1-8.

21. Fucentese SF, Tscholl PM, Sutter R, Brucker PU, Meyer DC, Koch PP. Bone autografting in medial open wedge high tibial osteotomy results in improved osseous gap healing on computed tomography, but no functional advantage: a prospective, randomised, controlled trial. Knee Surg Sports Traumatol Arthrosc 2019;27:2951-7.

22. Kim YT, Choi JY, Lee JK, Lee YM, Kim JI. Coronal tibiofemoral subluxation is a risk factor for postoperative overcorrection in high tibial osteotomy. Knee 2019;26:832-7. 